\title{
The distance of the centre of femoral head relative to the midline of the pelvis: a prospective $\mathrm{X}$-ray study of 500 adults
}

\author{
Yash B. Rabari*, Amol Sanap, D. V. Prasad, Krunal H. Thadeshwar
}

Department of Orthopaedic, Rural Medical Collage, LoniBk, Maharashtra, India

Received: 18 February 2017

Revised: 15 March 2017

Accepted: 24 March 2017

\author{
*Correspondence: \\ Dr. Yash B. Rabari, \\ E-mail: dryash.rabari@gmail.com
}

Copyright: ( $)$ the author(s), publisher and licensee Medip Academy. This is an open-access article distributed under the terms of the Creative Commons Attribution Non-Commercial License, which permits unrestricted non-commercial use, distribution, and reproduction in any medium, provided the original work is properly cited.

\begin{abstract}
Background: The mechanical axis of the femur is defined as the line joining the centre of the femoral head to the centre of the knee joint. One of the pre-requisites for a successful total knee replacement (TKR) is correct positioning of the implants, so that the mechanical axis of the limb is restored to neutral. During TKR surgery, the distal femoral anatomy can be visualized. However, to identify the mechanical axis of the femur, the location of the femoral head must be known.

Methods: We prospectively measured distance of centre of femoral head relative to the midline of the pelvis in 500 adults, using $\mathrm{x}$ ray of pelvic with both hip anteroposterior view done for medical causes during 2-May-2015 to 1-Jan2017 with satisfied the following inclusion and exclusion criteria. Patient gender and age were known. Both hips were clearly shown on the radiograph and not affected by any developmental or acquired condition that might deform normal anatomy. Radiographs demonstrating unacceptable pelvic tilt or rotation were excluded. Also, we excluded any cases where degenerative changes in the native hip were more severe than grade 1, based on the Tönnis classification.

Results: There were total 500 patients in which 250 were male and 250 were female. The mean age of male was 52.14 year ( $\mathrm{SD} \pm 80.80 \mathrm{~mm}, 95 \%$ CI 51.05 to $53.24 \mathrm{~mm}$ ) and female was 52.11 years (SD $\pm 8.82 \mathrm{~mm}, 95 \%$ CI 51.01 to $53.24 \mathrm{~mm}$ ). The mean distance of femoral head centre from midline in male was $95.02 \mathrm{~mm}$ (SD $\pm 2.20 \mathrm{~mm}, 95 \% \mathrm{CI}$ 94.75 to $95.30 \mathrm{~mm}$ ) and in female was $91.54 \mathrm{~mm}$ (SD $\pm 2.64 \mathrm{~mm}, 95 \%$ CI 91.22 to $91.87 \mathrm{~mm}$ ).

Conclusions: This study provide a useful information to determine the femoral head center relative to the midline of pelvis which useful intraoperatively.
\end{abstract}

Keywords: Femoral head, Pelvis

\section{INTRODUCTION}

The mechanical axis of the femur is defined as the line joining the centre of the femoral head to the centre of the knee joint. ${ }^{1}$ One of the pre-requisites for a successful total knee replacement (TKR) is correct positioning of the implants, so that the mechanical axis of the limb is restored to neutral. ${ }^{1-3}$ During TKR surgery, the distal femoral anatomy can be visualized. However, to identify the mechanical axis of the femur, the location of the femoral head must be known. Femoro-tibial malalignment in excess of $3^{\circ}$ causes eccentric mechanical loading through the implanted prostheses and is a recognized contributor of early mechanical failure. ${ }^{4,5} \mathrm{~A}$ fixed valgus cut angle of either $5^{\circ}$ or $6^{\circ}$, with respect to the anatomical axis of the femoral shaft, is frequently used in uncomplicated cases. This strategy uses the anatomical axis as a surrogate for the mechanical axis 
and relies upon a predictable angle between the two axes. However, this assumption has been shown to be inaccurate in at least $30 \%$ of patients. ${ }^{6}$ So that centre of femoral head plays an important role in total knee replacement surgery.

In the 1991 operation technique manual, for the use of Protek's F/S Modular TKR System, it was noted that "experience has shown that the average Caucasian hip is centred $9 \mathrm{~cm}$ from the midline". 7 This observation was tested by Freeman and Pinskerova, who reported the results of a study on 40 randomly selected computed tomography (CT) scans from the United Kingdom and 50 randomly selected Magnetic Resonance (MR) scans from the Czech Republic. ${ }^{8,9}$ They found that the mean distance from the pelvic midline to the centre of the femoral head was $8.9 \mathrm{~cm}$ in males and $8.8 \mathrm{~cm}$ in females.

Previous investigators have reported on the relation between the centre of the femoral head and the anatomy of the proximal femur. ${ }^{10,11}$ Although these investigators have demonstrated that landmarks of the proximal femur may be used to predict the position of the centre of the femoral head, such predictors are of little intra-operative value for TKR, as the bony anatomy of the proximal femur cannot be discerned by clinical palpation. ${ }^{11}$ Furthermore, variation in proximal femoral anatomy precludes the use of readily identifiable surface landmarks to predict the location of the centre of the femoral head. ${ }^{10,12}$ Other clinical methods used to identify the centre of the femoral head have included the topographical relationship of the latter with the ipsilateral femoral pulse or the anterior superior iliac spine (ASIS) and the use of ultrasound. ${ }^{13-15}$ With the use of CT scans, Mullaji et al recently reported that the centre of the femoral headlies at a mean of $7.9 \mathrm{~cm}$ and $8.1 \mathrm{~cm}$, for men and women, respectively, from the midline of the pelvis. ${ }^{16}$

\section{METHODS}

We prospectively measured distance of centre of femoral head relative to the midline of the pelvis in 500 adults, using $\mathrm{X}$ ray of pelvic with both hip anteroposterior view done for medical causes during 2nd May 2015 to $1^{\text {st }}$ January 2017.

Inclusion criteria were patients with known gender and age and whose both hips were clearly shown on the radiograph and not affected by any developmental or acquired condition that might deform normal anatomy.

Exclusion criteria were radiographs demonstrating unacceptable pelvic tilt or rotation and any cases where degenerative changes in the native hip were more severe than grade 1 , based on the Tönnisclassification. ${ }^{17,18}$

The radiographs had been obtained and stored digitally using picture archiving and communications system (PACS). Each image was analyzed independently by two investigators. The midline of the pelvis was identified as the longitudinal midline of the pubic symphysis. The perpendicular distance from the centre of the femoral head to the midline of the pelvis was measured (Figure 1). This is referred to as the femoral head centre to midline (FHC-M) distance.

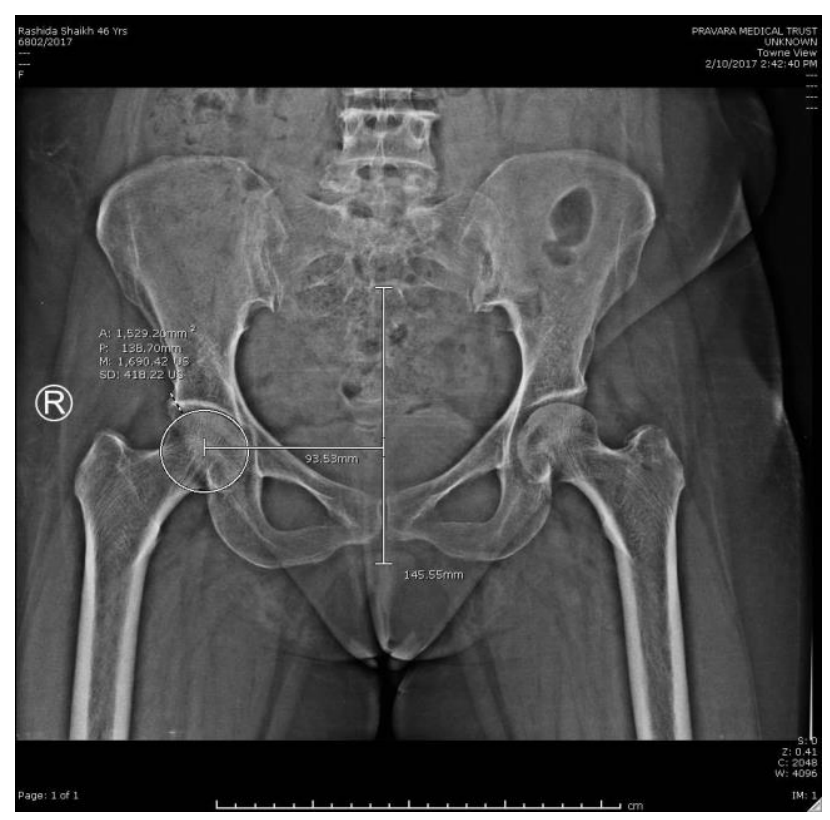

Figure 1: Method used to measure distance of FHC-M.

Statistical analyses were performed using Graph Pad Software, In. The relationship between variables was investigated using Pearson product-moment correlation coefficient (r). The independent-samples t-test was used to compare the FHC-M distance between the two genders. The paired-samples t-test and a Bland-Altman plot were used to assess the intra-observer reliability. ${ }^{19,20}$ Statistical significance was set at $\mathrm{p}<0.05$. Results are reported as mean \pm standard deviation, with $95 \%$ confidence interval $(\mathrm{CI})$.

\section{RESULTS}

There were total 500 patients in which 250 were male and 250 were female.

Table 1: Age of patients.

\begin{tabular}{|lll|}
\hline & No of patients & Mean age in years \\
\hline Male & 250 & $52.14(\mathrm{SD} \pm 8.80)$ \\
\hline Female & 250 & $52.11(\mathrm{SD} \pm 8.82)$ \\
\hline
\end{tabular}

Table 2: Distance of femoral head centre from midline of pelvis.

\begin{tabular}{|ll|}
\hline Gender & Mean $\mathrm{FHC}-\mathrm{M}$ distance in $\mathbf{~ m m}$ \\
\hline Male & $95.02(\mathrm{SD} \pm 2.20)$ \\
\hline Female & $91.54(\mathrm{SD} \pm 2.64)$ \\
\hline
\end{tabular}


The mean distance of femoral head centre from midline in male was $95.02 \mathrm{~mm}(\mathrm{SD} \pm 2.20 \mathrm{~mm}, 95 \%$ CI 94.75 to $95.30 \mathrm{~mm})$ and in female was $91.54 \mathrm{~mm}(\mathrm{SD} \pm 2.64 \mathrm{~mm}$, 95\% CI 91.22 to $91.87 \mathrm{~mm}$ ) (Table 2).

The difference between mean distance of femoral head centre from midline of male and female was $3.48 \mathrm{~mm}$ $(\mathrm{t}=16.03, \mathrm{df}=498,95 \% \mathrm{CI} 3.06$ to $3.90 \mathrm{~mm})$. The mean FHC-M distance was greater in men by $3.48 \mathrm{~mm}$ $(95.02 \pm 2.20 \mathrm{~mm}, 95 \%$ CI 94.75 to $95.30 \mathrm{~mm})$ than in women $(91.54 \pm 2.64 \mathrm{~mm}, 95 \% \mathrm{CI}, 91.22 \mathrm{~mm}-91.87$ $\mathrm{mm})$.

The mean FHC-M distance was measured by observer 1 as $93.21 \mathrm{~mm}(\mathrm{SD} \pm 2.97 \mathrm{~mm} \mathrm{95 \%} \mathrm{CI,} 92.84-93.56 \mathrm{~mm})$ and by observer 2 as $93.36 \mathrm{~mm}(\mathrm{SD} \pm 3.00 \mathrm{~mm} \mathrm{95 \%} \mathrm{CI}$, 92.98-93.73 mm). The mean difference between the two observers was $0.14 \mathrm{~mm}(\mathrm{t}=0.55,95 \% \mathrm{CI},-0.6729$ to $0.3776 \mathrm{~mm}$ ).

\section{DISCUSSION}

This is the study to demonstrate that the position of the centre of the femoral head relative to the midline of the pelvis. As expected, it varies with patients' gender. The mean difference between the genders is $3.48 \mathrm{~mm}$ $(\mathrm{t}=16.03, \mathrm{df}=498,95 \%$ CI 3.06 to $3.90 \mathrm{~mm})$. Although there was a statistical difference (paired-samples t-test) between the measurements of the two observers, the mean difference was only $0.14 \mathrm{~mm}(\mathrm{t}=0.55$, 95\% CI,0.6729 to $0.3776 \mathrm{~mm}$ ). We consider this to have little, if any, clinical significance. The difference is attributed to observer variation in the identification of the longitudinal line dividing the symphysis pubis and the identification of the centre of the femoral head.

In the study by Clark et al on the relationship of neck orientation to the shape of the proximal femur, the mean distance between the centre of the femoral head and the pubic symphysis was measured as $106.3 \mathrm{~mm}^{10} \mathrm{~A}$ possible explanation to this discrepancy is that Clark et al. undertook manual measurements, using traditional Xray films and the results may have been influenced by their calibration method. This hypothesis is supported by the findings of Pouget who reported on the offset and neck-shaft angle in THR in $2005 .^{21}$ Pouget measured the offset of the centre of rotation, defined as the distance between the centre of the femoral head and the pubic symphysis, in radiographs of 150 patients. He found a mean distance between the centre of the femoral head and the symphysis pubis of $90.5 \mathrm{~mm}$. This closely matches our results. $^{21}$

Previous investigators have attempted to localize the centre of the femoral head using body landmarks, such as the ASIS or the femoral pulse at the inguinal crease, and with use of ultrasound. ${ }^{13-15}$ A predictor model, constructed by Sugano, Noble and Kamaric, useful in locating the centre of the femoral head on a radiograph, would be of little practical use during TKR surgery. ${ }^{11}$ On the other hand, when performing a TKR, the pubic symphysis lends itself to easy palpation by the surgeon.

Recently, the distance between the pubic symphysis and the centre of the femoral head was measured with use of CT scans in 200 Indian adults. ${ }^{16}$ Although the mean reported difference between genders $(2.8 \mathrm{~mm})$ is similar to our findings, the mean FHC-M distance in that study was found to be $80 \pm 4 \mathrm{~mm}$. Both studies were undertaken on Caucasians so the discrepancy may be explained by difference in skeletal development during the growth years. Further comparative studies on individuals of different ethnic, social and cultural origins would throw further light on differences in skeletal development.

While our results suggest that using the gender-specific mean FHC-M distances (91.02 mm for females and 95.02 $\mathrm{mm}$ for males) results in a highly accurate estimation of the location of the centre of the femoral head in most patients, we do acknowledge some potential practical difficulties associated with the use of this method. The intra-operative localization of the pubic symphysis can be difficult in some patients (e.g. in morbid obesity). Furthermore, the super inferior location of the femoral head can be variable and does not necessarily coincide with the level of pubic symphysis. The method proposed in the present study should, therefore, be considered an adjunct to other reported techniques intended for intraoperative use. ${ }^{13}$

Our findings validate the senior author's assertion, first reported in his op-tech of 1991, that a point $9 \mathrm{~cm}$ lateral to the centre of the symphysis pubis could be used to predict the position of the centre of the femoral head in TKR surgery. ${ }^{7}$ While the clinical value of this knowledge may be lessened by the advent of computer-assisted navigation and CT/ MR-generated custom cutting blocks, surgeons who continue to use conventional instrumentation should find the information helpful.

\section{CONCLUSION}

This study provide a useful information to determine the femoral head centre relative to the midline of pelvis which useful intraoperatively.

\section{Funding: No funding sources Conflict of interest: None declared \\ Ethical approval: The study was approved by the institutional ethics committee}

\section{REFERENCES}

1. Kharwadkar N, Kent RE, Sharara KH, Naique S. 5 degrees to 6 degrees of distal femoralcut for uncomplicated primary total knee arthroplasty: is it safe? Knee. 2006;13:57-60.

2. Jiang CC, Insall JN. Effect of rotation on the axial alignment of the femur. Pitfalls in theuse of femoral 
intramedullary guides in total knee arthroplasty. Clin Orthop Relat Res. 1989:248:50-6.

3. Lotke PA, Ecker ML. Influence of positioning of prosthesis in total knee replacement. J Bone Joint Surg Am. 1977;59:77-9.

4. Bargren JH, Blaha JD, Freeman MA. Alignment in total knee arthroplasty. Correlated biomechanical and clinical observations. Clin Orthop Relat Res. 1983;(173):178-83.

5. Balakrishnan V, De SR, Lowe A. Radiographic assessment of alignment following TKA: outline of a standardized protocol and assessment of a newly devised trigonometric method of analysis. ANZ J Surg. 2010;80:344-9.

6. Bardakos N, Cil A, Thompson B, Stocks G. Mechanical axis cannot be restored intotal knee arthroplasty with a fixed valgus resection angle: a radiographic study. J Arthroplasty. 2007;22:85-9.

7. Freeman MAR, Samuelson KM. Freemansamuelson modular total knee replacement system. 1991.

8. Freeman MAR. Computers in TKR. Czech Society for Orthopaedics and Traumatology Meeting; Prague. 2006.

9. Pinskerova V, Sosna A, Pokorny D, Freeman MAR. A computerized tensor to assisttotal knee replacement. 10thNational Congress of the Czech Society for Orthopaedics and Traumatology; Znojmo, Czech Republic; 2006.

10. Clark JM, Freeman MA, Witham D. The relationship of neck orientation to theshape of the proximal femur. J Arthroplasty. 1987;2:99-109.

11. Sugano N, Noble PC, Kamaric E. Predicting the position of the femoral head centre. J Arthroplasty. 1999;14:102-7.

12. Theivendran K, Hart WJ. Is the tip of the greater trochanter a reliable reference for therotation centre of the femoral head in total hip arthroplasty? Acta Orthop Belg. 2009;75:472-6.
13. Sawant MR, Murty A, Ireland J. A clinical method for locating the femoral headcentre during total knee arthroplasty. Knee. 2004;11:209-12.

14. Ritter MA, Campbell ED. A model for easy location of the centre of the femoralhead during total knee arthroplasty. J Arthroplasty. 1988;3:59-61.

15. Matsuda Y, Ishii Y, Ichimura K. Identifying the center of the femoral head using ultrasonography to assess the higher accuracy of femoral extramedullary guides in TKA. J Orthop Sci. 2004;9(1):6-9.

16. Mullaji A, Shetty GM, Kanna R, Sharma A. Variability in the range of inter-anterior superioriliac spine distance and its correlation with femoral head centre. A prospective computed tomography study of 200 adults. Skeletal Radiol. 2010;39:363-8.

17. Clohisy JC, Carlisle JC, Beaule PE, Kim YJ, Trousdale RT, Sierra RJ, et al. A systematicapproach to the plain radiographic evaluation of the young adult hip. J Bone Joint Surg Am. 2008;90(4):47-66.

18. Tonnis D. Normal values of the hip joint for the evaluation of X-rays in children and adults. Clin Orthop Relat Res. 1976:(119):39-47.

19. Bland JM, Altman DG. Statistical methods for assessing agreement between two methods of clinical measurement. Lancet. 1986;1:307-10.

20. Petrie A. Statistics in orthopaedic papers. J Bone Joint Surg Br. 2006;88:1121-36.

21. Pouget G. Offset and neck-shaft angle in total hip arthroplasty: consequences. J Bone Joint Surg Br. 2005;90;243.

Cite this article as: Rabari YB, Sanap A, Prasad DV, Thadeshwar KH. The distance of the centre of femoral head relative to the midline of the pelvis: a prospective X-ray study of 500 adults. Int J Res Orthop 2017;3:565-8. 